(NASA-CR-200044) PREFERENTIAL HEATING OF LIGHT IONS OURING AN

N96-17861 IONOSPHERIC AR(+) INJECTION EXPERIMENT (NASA. Marshall SPace Flight (enter) $12 \mathrm{p}$

Unclas

$63 / 46 \quad 0098273$ 


\title{
Preferential heating of light ions during an ionospheric $\mathrm{Ar}^{+}$ injection experiment
}

\author{
C. J. Pollock, ${ }^{1}$ M. O. Chandler, ${ }^{1}$ T. E. Moore, ${ }^{1}$ R. L. Arnoldy, ${ }^{2}$ P. M. Kintner, ${ }^{3}$ \\ S. Chesney, ${ }^{3}$ and L. J. Cahill Jr. ${ }^{4}$
}

Abstract. The Argon Release for Controlled Studies (ARCS) 4 sounding rocket was launched northward into high altitude from Poker Flat Research Range on February 23, 1990. The vehicle crossed geomagnetic field lines containing discrete auroral activity. An instrumented subpayload released $100 \mathrm{eV}$ and $200 \mathrm{eV} \mathrm{Ar}{ }^{+}$ion beams sequentially, in a direction largely perpendicular to both the local geomagnetic field and the subpayload spin axis. The instrumented main payload was separated along field lines from the beamemitting subpayload by a distance which increased at a steady rate of $\sim 2.4 \mathrm{~m} \mathrm{~s}^{-1}$. Threedimensional mass spectrometric ion observations of ambient $\mathrm{H}^{+}$and $\mathrm{O}^{+}$ions, obtained on board the main payload, are presented. Main payload electric field observations in the frequency range $0-16 \mathrm{kHz}$, are also presented. These observations are presented to demonstrate the operation of transverse ion acceleration, which was differential with respect to ion mass, primarily during $100-\mathrm{eV}$ beam operations. The preferential transverse acceleration of ambient $\mathrm{H}^{+}$ions, as compared with ambient $\mathrm{O}^{+}$ions, during the second, third, fourth, and fifth $100-\mathrm{eV}$ beam operations, is attributed to a resonance among the injected $\mathrm{Ar}^{+}$ions, beam-generated lower hybrid waves, and $\mathrm{H}^{+}$ions in the tail of the ambient thermal distribution. This work provides experimental support of processes predicted by previously published theory and simulations.

\section{Introduction}

Large outward ion plasma flows from the low-altitude ionosphere into the near-Earth space provide much of the plasma which populates the Earth's magnetosphere. In the case of the heavy ions, these flows are not predicted by cold hydrodynamic models, such as the polar wind models of Banks and Holzer [1969]. Large-scale acceleration of heavy ions from energies characteristic of ionospheric plasma temperatures (fraction of an $\mathrm{eV})$ to escape energy $(\sim 10 \mathrm{eV}$, in the case of $\left.\mathrm{O}^{+}\right)$is required to account for observed heavy ion outflows. Transverse ion acceleration (TIA) in the topside ionosphere, followed by outward acceleration under the influence of the mirror effect in the diverging high-latitude geomagnetic field geometry, occurs commonly at auroral latitudes. Determination of the precise mechanisms responsible for this TIA has been the focus of a large body of research since early in situ observations of topside ionospheric TIA were reported by Sharp et al. [1977] and Klumpar [1979].

\footnotetext{
${ }^{1}$ NASA Marshall Space Flight Center, Space Plasma Physics Branch, Space Sciences Laboratory, Huntsville, Alabama.

${ }^{2}$ Institute for the Study of Earth, Oceans, and Space, University of New Hampshire, Durham.

${ }^{3}$ Department of Electrical Engineering, Cornell University, Ithaca, New York.

${ }^{4}$ School of Physics and Astronomy, University of Minnesota, Minneapolis.
}

Copyright 1995 by the American Geophysical Union.

Paper number $94 \mathrm{JA03238.}$

0148-0227/95/94JA-03238\$05.00
In the Argon Release for Controlled Studies (ARCS) program, we have sought to make progress in understanding space plasma wave-particle interactions and charged particle acceleration processes through the use of controlled argon ion beam injections from suborbital rocket platforms. In each but the first of these four flights, a separated subpayload has housed one or more ion beam generators (ARCS-1 was composed of an integral payload with no separable subpayload). These beam generators operate under program control as the subpayload and main payload move apart, across and along local geomagnetic field lines. Plasma diagnostics have been obtained locally on the emitting subpayload and at locations remote from the injection site (subpayload) using main payload instruments.

Injection of the ion beam from the subpayload provides a known momentum and energy source for the local plasma. The beam is inherently spatially localized and diverges from the injection point as it gyrates about the local geomagnetic field. There are large gradients in the beam density and flux, particularly in the direction transverse to the beam velocity, although also along the beam path. The beam density and gradients are largest near the emitting payload.

Transverse injection of the heavy ion beam is expected to produce local electric fields and currents in the background plasma. Steady state emission of ions from the beam payload requires current balance at the payload. This may be provided by electron emission or by absorption of ambient ions. Emitted electrons will be more strongly magnetized than beam ions due to their much smaller mass. These electrons cannot propagate across field lines with the ions in the absence of a polarization electric field oriented perpendicular to both the beam velocity and the local magnetic field. If such an electric field 
is not established, emitted electrons are constrained to flow away from the beam payload along magnetic field lines. In this case, the ion beam itself is neutralized by weak electron currents flowing along magnetic field lines connected to the beam, and the emitting payload is neutralized by rather intense electron currents flowing along field lines connected to the immediate vicinity of the beam payload.

These macroscopic effects, as well as microscopic processes, such as resonant interactions between beam ions and background ions, have the potential to significantly modify the emitted beam distribution and the ambient plasma distributions. In this paper we will focus on the observation of interactions between the beam $\mathrm{Ar}^{+}$ions and ions in the background plasma. These interactions are mediated by oscillating elec tric fields within the background plasma. In the case of the ARCS -4 , these interactions fall into several categories. Close to the beam-emitting payload, a strong interaction between the background plasma and the $100-\mathrm{eV}$ beam was observed to produce extensive modification of the distributions of all observed ambient ion species. At intermediate distances from the beam payload, the interaction was weaker and only the background $\mathrm{H}^{+}$ions were significantly modified, being heated in the direction transverse to $\mathbf{B}$, primarily during $100-\mathrm{eV}$ injections. At intermediate distances, $200-\mathrm{eV}$ injections produced only slight modification of the $\mathrm{H}^{+}$and had no measurable effect on the $\mathrm{O}^{+}$. At large distances from the emitting payload, no significant modifications of the ambient plasma distributions were observed. We will attempt to place these observations and corresponding plasma wave observations in context with the works of Scales and Kintner [1990a, b], in which interactions between a heavy ion beam and the ionospheric plasma were studied using linear Vlasov theory and particle in cell (PIC) simulations.

\section{Previous Related Work}

Several important ion beam injection experiments have been conducted using sounding rockets. The first major exper imental effort was the Porcupine project [Haerendel and Sagdeev, 1981; Häusler et al., 1986], conducted in 1979. This experiment featured a $200-\mathrm{eV} \mathrm{Xe} \mathrm{Xe}^{+}(\mathrm{m} / q=130)$ beam emitted across field lines from a spinning subpayload which was separated across magnetic field lines from the main payload and other instrumented subpayloads. During this experiment, plasma wave emissions at frequencies near multiples of the local $\mathrm{H}^{+}$gyro frequency were observed [Jones, 1981; Kintner and Kelley, 1981]. These were interpreted as Bernstein mode emissions due to a resonant interaction between the emitted $\mathrm{Xe}^{+}$ions and local hydrogen ions traveling near the same speed. Only limited charged particle observations were available from Porcupine and the question of ambient thermal ion heating was not addressed, although those experimenters did conclude that ambient electron heating to energies up to 20 $\mathrm{eV}$ was induced by the injected $\mathrm{Xe}^{+}$beam.

The ARCS series of experiments have all used injection of $\mathrm{Ar}^{+}$ions $(\mathrm{m} / q=40)$ into the topside ionospheric plasma. All but the first of these missions (ARCS-1) have featured a separable subpayload which housed one or more $\mathrm{Ar}^{+}$beam generators. The subpayloads were ejected generally along (either up or down) magnetic field lines from the instrumented main payload. In each of these cases, the subpayload carried a minor complement of instruments, while the main payload was more fully instrumented.
In each of the three previous ARCS experiments, a variety of plasma wave emissions were observed in response to the injected ion beam. Reports of beam-induced plasma wave observations from ARCS-3 have been presented by Erlandson [1986] and Erlandson et al. [1987, 1989]. Pollock [1987] and Pollock et al. [1988] have presented detailed ion observations obtained from the ARCS-3 mission, although no massdiscriminated measurements were available. The ARCS-3 observations, obtained using a capped hemisphere imaging spectrometer and verified with octospheric ion energy analyz ers, included a persistent population of $90^{\circ}$ pitch angle ions, extending in energy from $5 \mathrm{eV}$ to $60 \mathrm{eV}$, present when the beam was on. It is not known whether these were energized ambient ions or degraded beam ions.

To our knowledge, the most detailed theoretical studies of the stability of injected ion beams have been conducted by Scales and Kintner [1990a, b]. In the first of these companion papers [Scales and Kintner, 1990a], the authors built on previous work in providing a detailed picture of the development of several relevant linear instabilities, focusing on interactions between a heavy ion beam and a background plasma, typically $\mathrm{O}^{+}$dominated, with $\mathrm{H}^{+}$as a minor species. They treated the lower hybrid instability in both the fluid and kinetic limits, defining a beam-plasma coupling parameter

$$
\tau^{2} \equiv \frac{\omega_{b}^{2}}{\omega_{o}^{2}+\omega_{H}^{2}} \frac{v_{d}^{2}}{v_{b}^{2}},
$$

where $\omega_{(\mathrm{H} y, O y)}$ is the ion species plasma frequency and $v_{d}$ and $v_{b}$ are the beam drift and thermal velocities, respectively. Scales and Kintner demonstrated that for $\tau^{2}>1$, corresponding to large fractional beam density and/or large beam drift to thermal velocity ratio, the beam-plasma coupling is strong. In this case, broadband waves are generated due to the dominant fluid lower hybrid instability, at frequencies extending up to the local lower hybrid frequency. For the case $\tau^{2}<1$, the beam is weakly coupled to the plasma, and wave growth is due to the kinetic lower hybrid instability and is confined to frequencies near and just below the local lower hybrid frequency.

The lower hybrid instabilities result from a treatment of the background ion plasma as unmagnetized (the heavy beam ions are typically considered unmagnetized). When including the effects of the geomagnetic field, gyro harmonic instabilities are shown to become important for $\tau^{2}<1$ and beam drift velocities of the order of the local ion thermal velocity. Specifically, they showed that for low beam densities and beam drift velocities up to several times the $\mathrm{H}^{+}$thermal velocity, the $\mathrm{H}^{+}$gyro harmonic instability dominates and produces electrostatic emissions in bands typically located immediately below multiples of the $\mathrm{H}^{+}$gyrofrequency. Here, Scales and Kintner [1990a] have made contact with one of the more common spectral features observed in the beam injection experiments.

In their second paper, Scales and Kintner [1990b] went on to study the nonlinear behavior of the beam-plasma systems by conducting PIC simulations. This study supported many of their conclusions based on the linear analysis. The simulation study also permitted investigation of the response of the background plasma ions to the beam injection. They found that background transverse ion heating was the dominant wave growth saturation mechanism for the warm beams relevant to the rocket-borne beam experiments. The broadband lower 
hybrid instability was primarily responsible for the simulated transverse ion heating. The background $\mathrm{H}^{+}$was more extensively heated than the $\mathrm{O}^{+}$. This is due to the following:

1. Since the simulation beam drift velocity was several times larger than the $\mathrm{H}^{+}$thermal velocity, the resulting broadband waves had phase velocities also larger than the $\mathrm{H}^{+}$ thermal velocity and were therefore more closely in resonance with $\mathrm{H}^{+}$ions than the slower $\mathrm{O}^{+}$ions.

2. Since the (unmagnetized) trapping energy of the $\mathrm{O}^{+}$is larger than that of the $\mathrm{H}^{+}$by the ratio $\left(\mathrm{m}_{\mathrm{O}}^{+} / \mathrm{m}_{\mathrm{H}}{ }^{+}\right.$), larger amplitude waves were required to trap and energize the $\mathrm{O}^{+}$.

Scales and Kintner [1990b] also observed simulated $\mathrm{O}^{+}$ heating; however, larger beam densities were required such that the coupling parameter $\tau^{2}$ was of the order of 1 . The larger beam densities produced larger amplitude waves, capable of trapping the massive $\mathrm{O}^{+}$ions. At the largest beam drift velocities, neither the $\mathrm{H}^{+}$nor $\mathrm{O}^{+}$thermal velocities are large enough for the ions to resonate with beam-generated waves and neither species showed significant heating. They also observed modest parallel electron heating due to finite $k_{\mathbf{l}}$.

\section{ARCS-4 Experiment}

ARCS-4 is the most recent of the ARCS series of ionospheric ion beam injection experiments. It was launched from the Poker Flat Research Range on February 23, 1990, at 073125 GMT. The payload traveled northward toward an apogee of $614 \mathrm{~km}$ at $443 \mathrm{~s}$ elapsed time after launch (TAL). The ARCS-4 payloads passed over an intense auroral arc after apogee, near $580 \mathrm{~s}$ TAL, at altitudes near $540 \mathrm{~km}$. Observations of the plasma environment associated with this auroral form will be reported elsewhere (T. E. Moore et al., Plasma heating and flow in an auroral arc, submitted to Journal of Geophysical Research, 1994; hereafter referred to as Moore et al., submitted manuscript). The subpayload was separated from the main payload at $144 \mathrm{~s}$ TAL. It was deployed upward, along the magnetic field with a fieldaligned speed of approx imately $2.4 \mathrm{~m} \mathrm{~s}^{-1}$ and cross-field speed of near $0.1 \mathrm{~m} \mathrm{~s}^{-1}$. The payloads and deployment geometry are illustrated in Figure 1. Plate 1a shows the altitude trajectory of the ARCS-4 payloads.

The separated subpayload carried a single $\mathrm{Ar}^{+}$beam generator. This source emitted approximately $100 \mathrm{~mA}$ of $\mathrm{Ar}^{+}$ions in a $60^{\circ}$ cone centered on the direction perpendicular to the subpayload spin axis. Since this axis was nearly aligned with the magnetic field, the ion emissions were centered nearly perpendicular to $\mathbf{B}$, providing a cross-field injection scenario. The gun was operated under program control. This sequence is illustrated in Plate 1b. The typical sequence was gun-on (100 eV), $12 \mathrm{~s}$; gun-off, $10 \mathrm{~s}$; gun-on $(200 \mathrm{eV}), 12 \mathrm{~s}$; gun-off, $10 \mathrm{~s}$.

Note from Plate $1 \mathrm{~b}$ that there were three significant deviations from the above sequence. The first gun firing commenced near $162 \mathrm{~s}$ after launch and lasted for $20 \mathrm{~s}$. Second, the gun program showed an anomaly near $421 \mathrm{~s}$ after launch. At that time, a $200-\mathrm{eV}$ firing event (414-427 s) was in progress when the anode voltage changed from 200 volts to 100 volts (see Plate $1 \mathrm{~b}$ ). The gun then turned off at $427 \mathrm{~s}$, as planned, but the following beam event, scheduled to be 100 $\mathrm{eV}$, operated at $200 \mathrm{eV}$. From that time, the actual beam energies were reversed from those intended. Finally, the last three beam firings were all $200-\mathrm{eV}$ events, whereas one of these (beginning near $710 \mathrm{~s}$ after launch) should have been at $100 \mathrm{eV}$. It is also noted that the anode voltage measured dur-

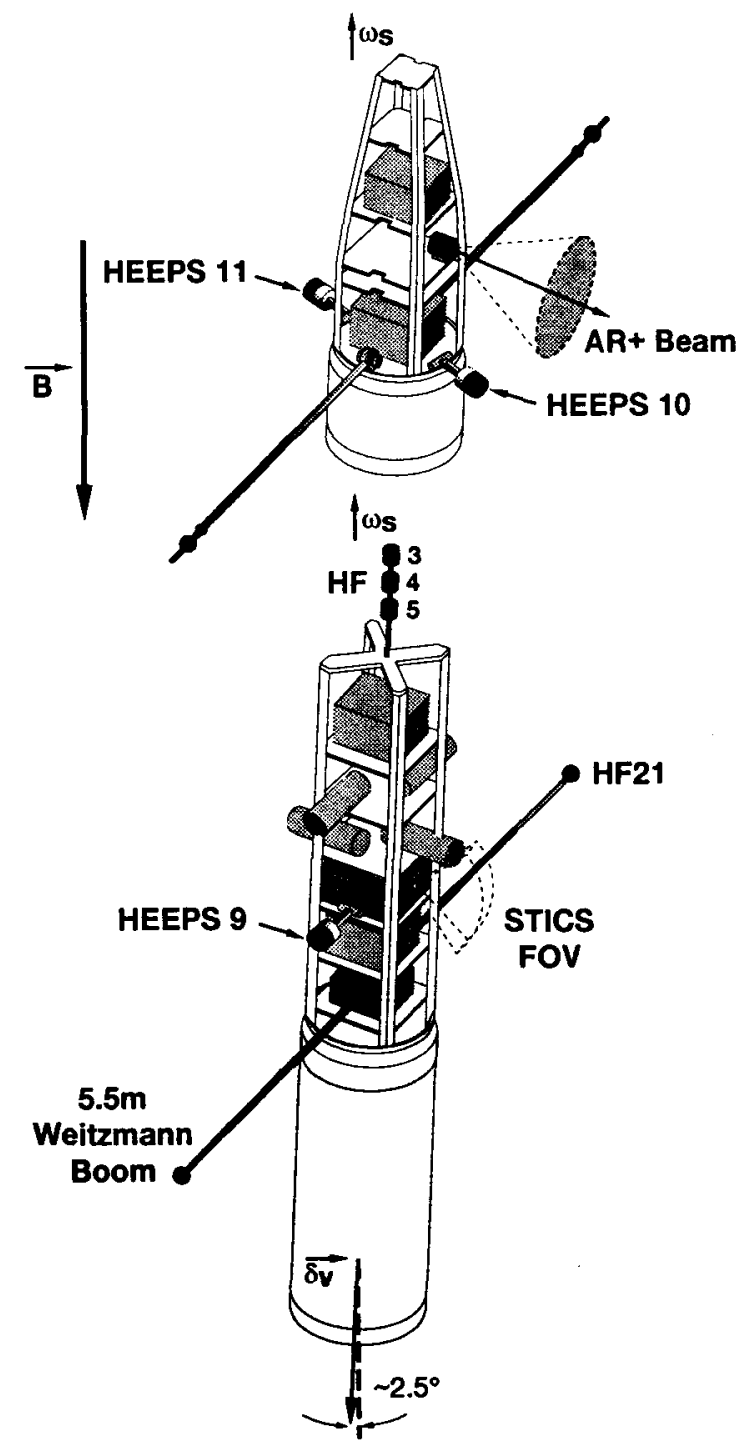

Figure 1. Schematic illustration of the Argon Release for Controlled Studies ARCS-4 experiment and the relative geometry of the two payloads as they were separated in flight are shown.

ing gun-off periods drifted upward during the flight. While this did not affect the operation of the gun, it may have affected the electrostatic environment of the subpayload.

$\mathrm{Ar}^{+}$ions emitted by the gun were measured throughout the flight, using both the main payload-mounted superthermal ion composition spectrometer (STICS), and hemispheric electrostatic energy and pitch angle spectrometer (HEEPS) sensors mounted on both payloads. Those measured at the subpayload using HEEPS-10 are shown in Plate 1c. This plot shows the raw nonmass-discriminated ion count rate in energy-time greyscale spectrogram format. The beam ions exciting the response shown here have traversed at least one magnetic gyration between emission and detection. Their temporal signature and alternating maximum energies near $100 \mathrm{eV}$ and $200 \mathrm{eV}$ reflect the ion gun operation as described above and shown in Plate $1 \mathrm{~b}$. Note the reduction in HEEPS thermal ion count rates at the subpayload when the gun fires. This may be due to positive subpayload charging. Kaufmann et al. [1989] discussed negative charging of the emitting payload due to the 


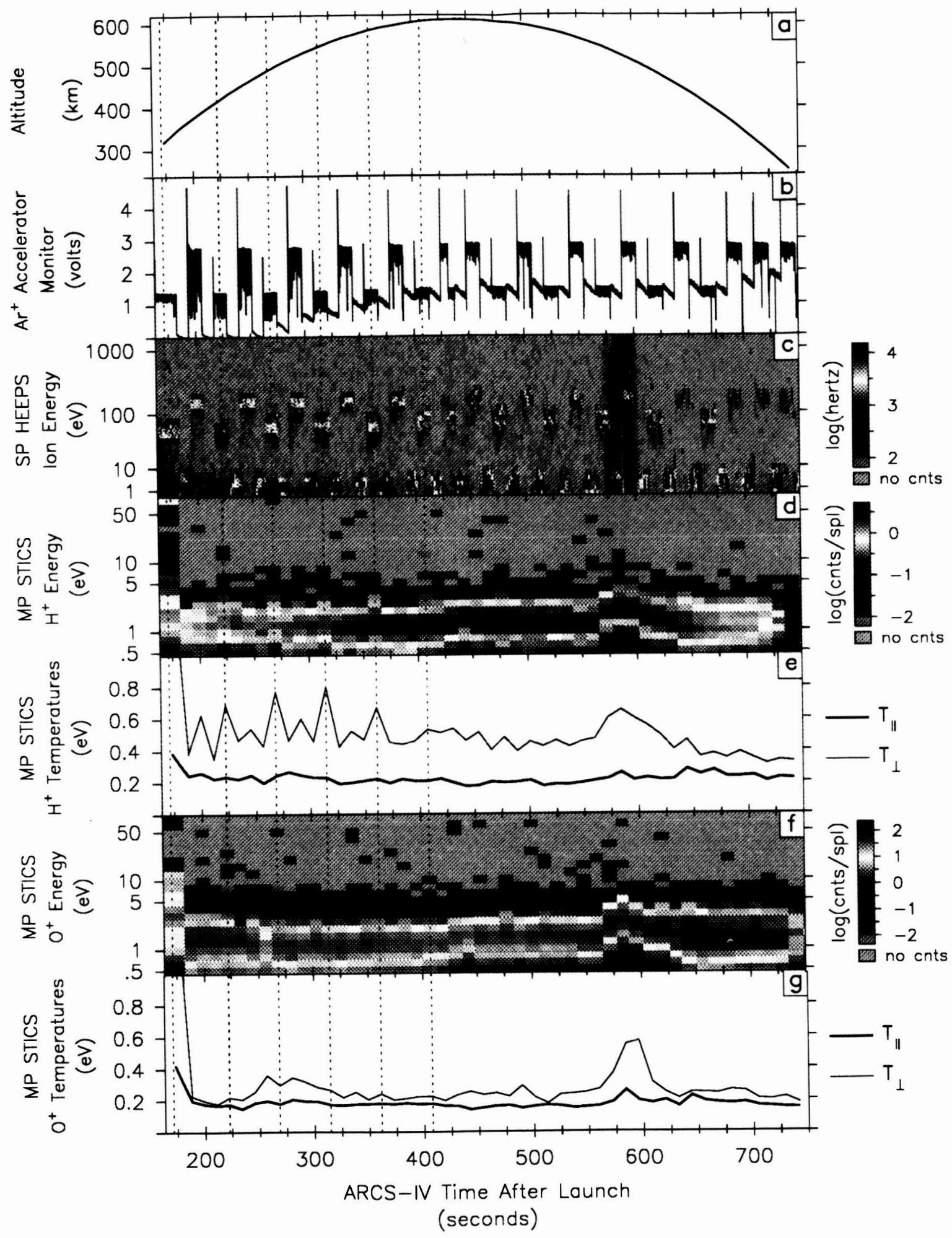

Plate 1. Flight summary of the ARCS-4 experiment. All quantities are plotted versus time after launch along the horizontal axis. Dashed vertical lines extending from the top to bottom of the plate indicate the times of the first six 100-eV beam events. (a) The measured altitude of the ARCS-4 payloads is shown. (b) Monitor voltage proportional to the accelerating voltage applied to the ion gun is shown. Values of $\sim 1.5 \mathrm{~V}$ $(\sim 3 \mathrm{~V})$ correspond to beam energies of $100 \mathrm{eV}(200 \mathrm{eV})$; (c) Energy-time spectrogram of hemispheric electrostatic energy and pitch angle spectrometer (HEEPS)-10 ion count rate measured at the subpayload is shown. (d) Energy-time spectrogram of superthermal ion composition spectrometer (STICS) $\mathrm{H}^{+}$count rates measured at the main payload is shown. (e) Parallel $\left(\mathrm{T}_{\|}\right)$and perpendicular $\left(\mathrm{T}_{\perp}\right)$ hydrogen ion temperatures derived as integral moments of the measured three-dimensional $\mathrm{H}^{+}$velocity distributions are shown. (f) Energy-time spectrogram of STICS $\mathrm{O}^{+}$count rates measured at the main payload is shown. (g) Parallel $\left(\mathrm{T}_{\|}\right)$ and perpendicular $\left(T_{\perp}\right)$ oxygen ion temperatures derived as integral moments of the measured threedimensional $\mathrm{O}^{+}$velocity distributions are shown. 
inability of magnetized gun electrons to escape the payload. Possible positive charging of the ARCS-4 subpayload will be the subject of a future study. The ion observations shown in Plates 1d-1g will be discussed below.

The STICS instrument was used to acquire the massresolved positive ion data presented in this paper. A brief description of the STICS instrument is provided by Moore et al. (submitted manuscript). This instrument electrostatically sweeps polar angle (with respect to payload spin axis), energy per charge, and mass per charge (via electrostatic acceleration into a magnetic sector spectrometer) within nested program loops. The polar angle sweep provided coverage of $\pm 70^{\circ}$ with respect to the vehicle spin axis and therefore approximately $20^{\circ}-160^{\circ}$ in magnetic pitch angle, due to the near antialignment between the spin axis and the geomagnetic field. Polar angle, energy, and mass were swept in the inner, middle, and outermost program loops, respectively. On ARCS 4 , the time required to execute a complete sweep sequence was $1.54 \mathrm{~s}$. The third observational dimension (azimuthal angle) was obtained through spacecraft spin. In this way, a complete three-dimensional measurement of the species distribution function was acquired approximately every $12 \mathrm{~s}$, which is the beat period between the $1.54 \mathrm{~s}$ voltage control loop and the $1.77 \mathrm{~s}$ payload spin. Analysis of the STICS ion data has been synchronized with the beam on-off cycle, so that the STICS data presented here are the result of acquisition of three-dimensional velocity distributions, acquired over each of the ion gun-on or gun-off intervals, typically 10-12 s. For the shorter of these intervals, typically gun-on periods, the three-dimensional velocity space remains approximately $15 \%$ undersampled.

\section{Observations}

\section{Flight Overview}

ARCS-4 wave and positive ion observations obtained aboard the main payload are presented on a flight summary basis and also for selected flight times and ion beam modes. As described above, accumulation of the STICS ion data has been synchronized with the beam-on/beam-off cycle, so that available mass resolved data are the result of acquisition of three-dimensional velocity distributions, averaged over indi vidual ion gun-on or gun-off intervals. When presenting STICS flight summary data (as in Plates Id through $1 \mathrm{~g}$ ), the data are organized in this way, with one point in time for each distinct ion gun state. However, due to the undersampling described above and to improve statistics, when presenting distribution functions (as in Plates 2 and 3) or energy spectra (as in Figure 2), STICS data from more than one like beam interval (100$\mathrm{eV}, 200-\mathrm{eV}$, or off) spaced closely in time have been combined, as indicated in the figures and captions.

Panels $1 \mathrm{~d}$ and $1 \mathrm{f}$ show energy-time spectrograms of the $\mathrm{H}^{+}$ and $\mathrm{O}^{+}$count rates for the entire flight. The plotted values were obtained by collapsing the three-dimensional count rate distributions over the two angular coordinates. These two spectrograms show similar energy distributions throughout the flight for the two species. The peak in the count rates occurs in the range $1-2 \mathrm{eV} / q$. The decreasing count rate below the thermal peak may be attributed to a nonzero positive spacecraft potential. The maximum $\mathrm{O}^{+}$count rates peak on the upleg and downleg, and the maximum $\mathrm{H}^{+}$rates peak near apogee, reflecting normal geophysical density trends with alti- tude for these two ion species. Near $600 \mathrm{~s}$ TAL, a broad energy enhancement is observed in both species, coincident with passage through a band of intense auroral activity. The ion heating observed within this event is the subject of another paper (Moore et al., submitted manuscript).

The focus of this report is on the narrow bands of extended energy response seen in the $\mathrm{H}^{+}$and $\mathrm{O}^{+}$near $160 \mathrm{~s}$ TAL and in the $\mathrm{H}^{+}$near $220,270,315,360$, and $405 \mathrm{~s}$ TAL. Each of these localized enhancements coincides with times that the ion gun was operating in the $100-\mathrm{eV}$ mode on the subpayload, above (indicated by dashed vertical lines from the top to bottom of each panel in Plate 1). The enhancement near $160 \mathrm{~s}$ is due to the first $100-\mathrm{eV}$ event when the two payloads were quite close to each other. The others occur at larger separation distances at which the beam plasma coupling is evidently not as strong. Note that these enhancements are only noticeable in the $\mathrm{O}^{+}$ during the first $100-\mathrm{eV}$ event and fade out in the $\mathrm{H}^{+}$after the fifth or sixth $100-\mathrm{eV}$ event. They are not evident in either species when the gun is emitting $200 \mathrm{eV}$ ions, with the exception of the first $200-\mathrm{eV}$ event, near $200 \mathrm{~s}$ TAL.

\section{Detailed Positive Ion Observations}

Plates $1 \mathrm{e}$ and $1 \mathrm{~g}$ provide a more quantitative view of the ion heating produced by the beam. Here we present the parallel and perpendicular temperatures of the $\mathrm{H}^{+}$(Plate le) and $\mathrm{O}^{+}$(Plate 1g) as functions of time throughout the flight. These temperatures are derived as integral moments over the threedimensional velocity distribution of each species, as measured by STICS. It is noted that the measured background ion perpendicular temperatures are typically larger by a factor of 2-3 than the corresponding parallel temperatures, except at the lower altitudes. It may be that the increase in parallel temperatures with decreasing altitude and the persistent temperature anisotropy in both species are analysis artifacts, arising in part from an interaction between the effects of spacecraft ram and instrument blind spots near the fieldaligned and antifield-aligned directions (note that these moments have been computed without any attempt to model the unsampled phase space densities within the instrument blind spots). These effects will be the subjects of future study. The effect of the aurora on the ion temperatures is evident near $600 \mathrm{~s}$ TAL.

The vertical lines in Plate 1 indicate times that the subpayload plasma gun was firing in the $100-\mathrm{eV}$ mode. Attention is directed to the series of enhancements (by factor of 2 ) in the perpendicular $\mathrm{H}^{+}$ion temperature, at times when the $100-\mathrm{eV}$ ion beam was on during the first through fourth $100-\mathrm{eV}$ firings, in particular. There are also enhancements in the $\mathrm{H}^{+}$perpendicular temperature when the $200-\mathrm{eV}$ beam was on, but these are more modest, except for the first 200-eV event (near $200 \mathrm{~s}$ TAL) when the two payloads were still quite close together and the $\mathrm{H}^{+}$perpendicular temperature increased to $0.9 \mathrm{eV}$. By contrast, with the exception of the first $100-\mathrm{eV}$ beam event, the $\mathrm{O}^{+}$temperature shows no significant sensitivity to the operation of the beam. The first $100-\mathrm{eV}$ beam event is different from the rest. More extreme $\mathrm{H}^{+}$heating is observed in this case, and the $\mathrm{O}^{+}$is seen to undergo even more intense perpendicular heating than the $\mathrm{H}^{+}$, reaching a bulk temperature of $3 \mathrm{eV}$ !

As mentioned above, the temperatures presented in Plate 1 were obtained through integration of the measured species three-dimensional velocity distributions. Color-coded, two- 

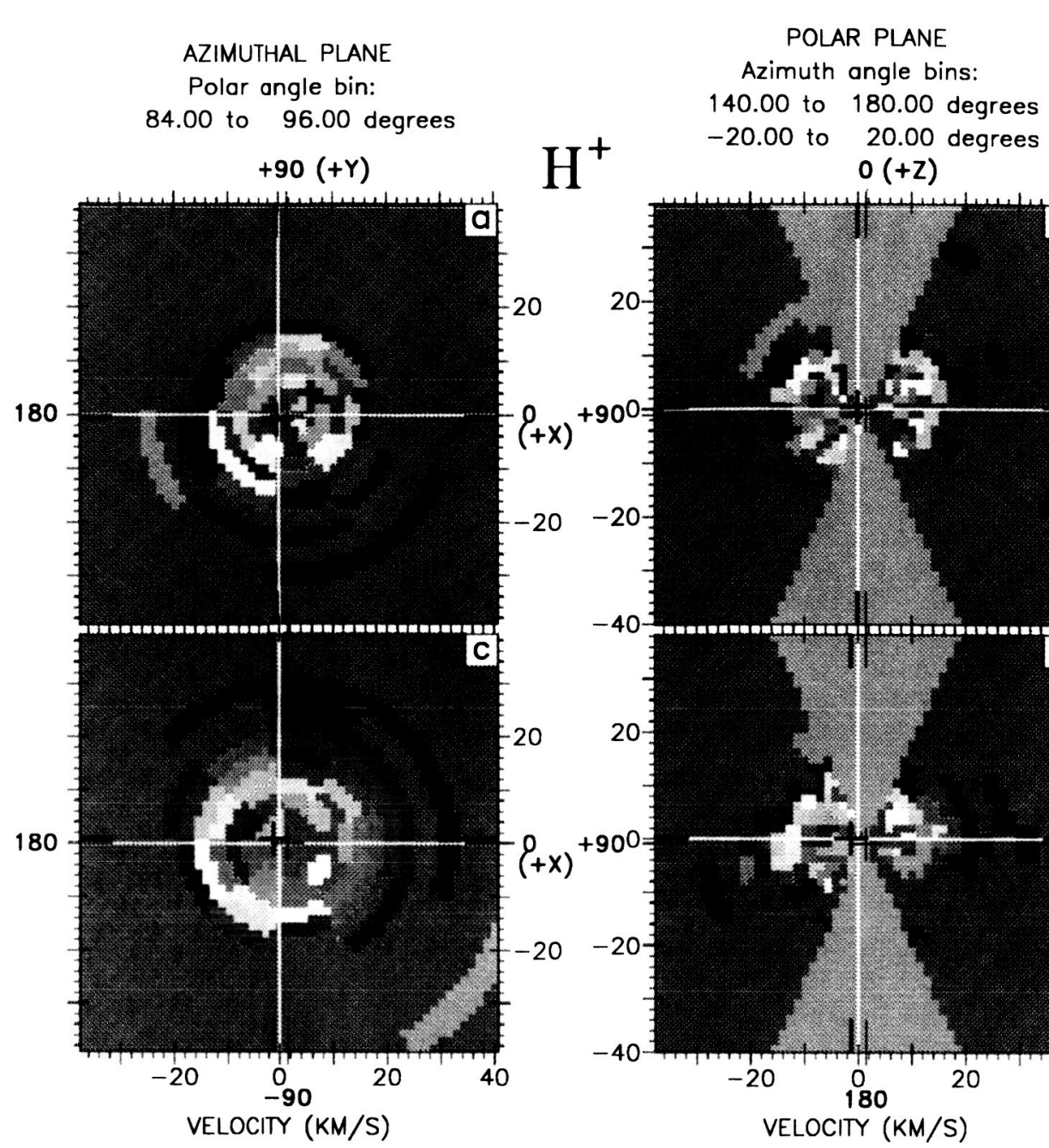

POLAR PLANE

Polar angle bin:

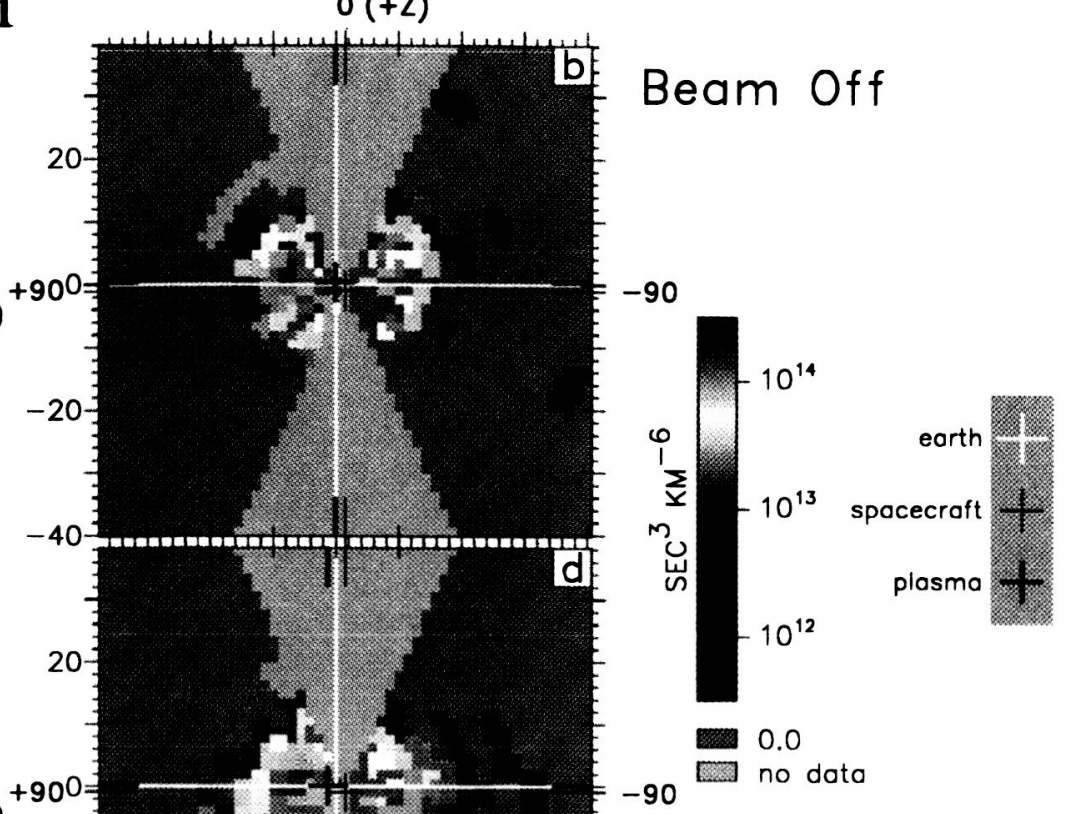

Plate 2. Three-dimensional representations of the $\mathrm{H}^{+}$velocity distribution for (a-b) gun-off and (c-d) 100 $\mathrm{eV}$, gun-on periods during the ARCS-4 flight. Each pair results from averaging the data from several like intervals. The gun-off data are from gun-off intervals following the second $200-\mathrm{eV}$, third $100-\mathrm{eV}$, and third $200-\mathrm{eV}$ events. The $100-\mathrm{eV}$ gun-on data are from the third and fourth $100-\mathrm{eV}$ events. Each data point is represented by a color-coded value of the conventional phase space density, arrayed in two panels representing cuts through the full velocity distribution (left) in the plane perpendicular to the local magnetic field and (right) in a plane containing the local magnetic field. The azimuth of the cut in the right-hand panels is chosen so as to cut through the maximum of the velocity distribution. The light, medium, and heavy cross hairs show the location of the Earth, spacecraft, and plasma frames of reference, respectively.

dimensional cuts of the three-dimensional $\mathrm{H}^{+}$and $\mathrm{O}^{+}$distributions functions are presented in Plates 2 and 3, respectively. The format for these data displays is the same for both plates. The top panels show data obtained when the $\mathrm{Ar}^{+}$beam was off. The bottom panels show data obtained when the beam was firing in the 100-eV mode. On the left in Plates 2 and 3 are shown azimuthal ion distributions, with B (downward) directed out of the page, while on the right, the values of the distribution near the $x-z$ plane (containing both spacecraft velocity and geomagnetic field) are shown. Values of the distribution function are coded as indicated in the color bar at the right. Each cut results from averaging the measurements from several three-dimensional distributions together in order to maximize data coverage and improve the statistics. These periods have been indicated on the displays and in the figure captions. Regions of missing data near the magnetic field directions are due to limitations on the electrostatic sweep of the instrument look direction. We don't fully understand the presence of zero valued data at small $\mathrm{H}^{+}$speeds. This could be associated with our estimates of spacecraft potential $(-0.7 \mathrm{~V}$ and $-0.8 \mathrm{~V}$ for the gun-off and gun-on periods shown), which were based primarily on the $\mathrm{O}^{+}$data. The views of the distributions provided in Plates 2 and 3 represent the most complete information presentable, regarding the observed ion distributions. The $\mathrm{H}^{+}$ions of Plate 2 show heating when the $100-\mathrm{eV}$ beam is on (Plates $2 \mathrm{c}$ and $2 \mathrm{~d}$ ), as compared to the ambient state (Plates $2 a$ and $2 b$ ). Further, it is clear that this heating is in the transverse (to B) direction. By contrast, the $\mathrm{O}^{+}$data shown in Plate 3 show no significant difference between the gun-on and gun-off data.

In Figure 2, three-dimensional data arrays for the two species have been collapsed into a single dimension to 

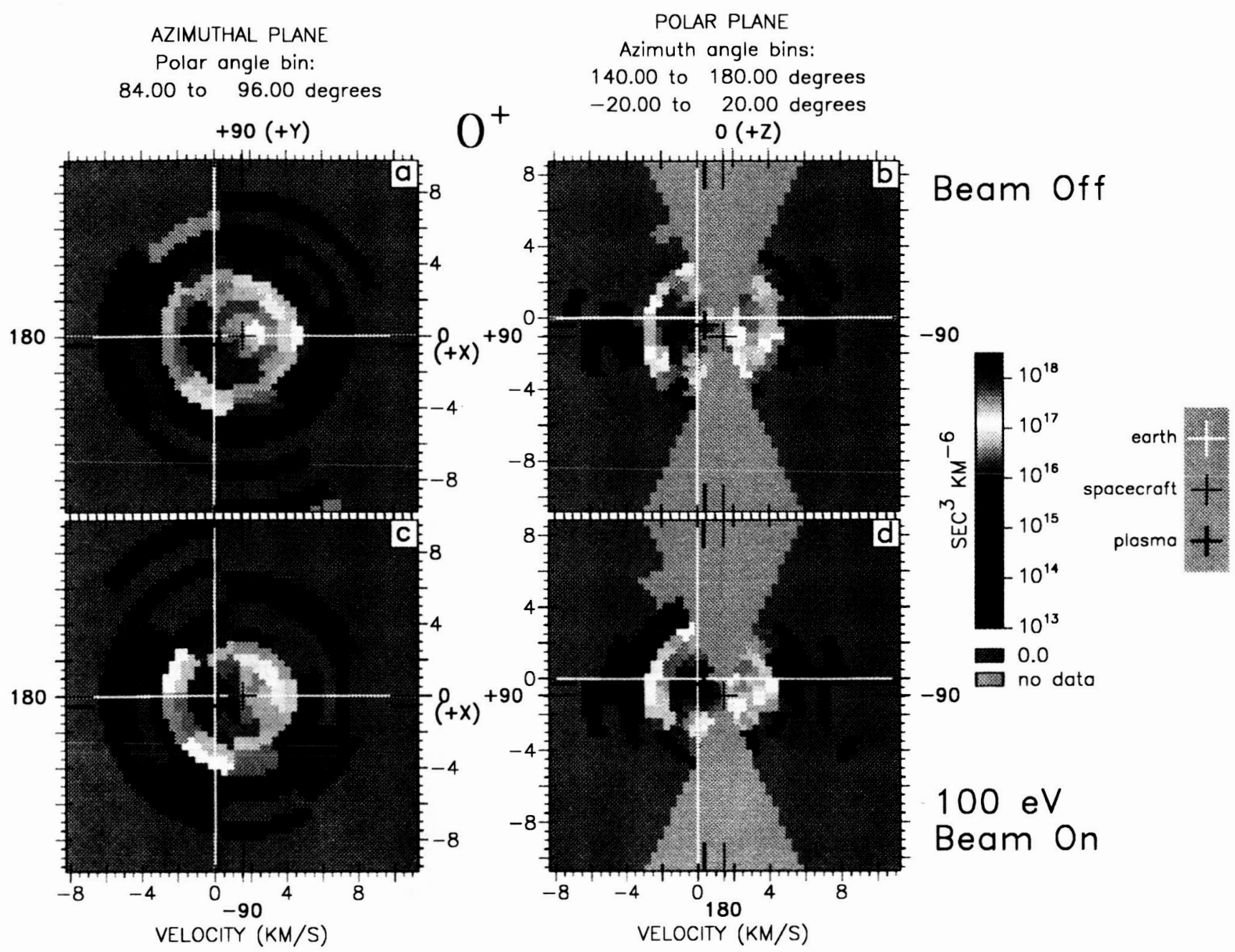

Plate 3. Same as Plate 2 but for $\mathrm{O}^{+}$.

display the species energy spectra. In this case, we have combined $90^{\circ}$ pitch angle ions with the two neighboring pitch angles (centered near $78^{\circ}$ and $102^{\circ}$ ) and included all magnetic azimuth angles for several sets of experimental condi tions. Here the one-dimensional $\left(V_{\text {perp }}\right) \mathrm{O}^{+}$and $\mathrm{H}^{+}$distribution functions measured when the beam was on (filled symbols) during the first firing of the $100-\mathrm{eV} \mathrm{Ar}^{+}$beam (Figure 2a), the third and fourth 100-eV beam firings (Figure $2 \mathrm{~b}$ ), and the second and third 200-eV beam firings (Figure 2c) are displayed for comparison alongside those obtained when the beam was off (unfilled symbols). The beam-off data are the same in all three panels and were acquired by averaging measurements made during gun-off intervals following the second $200-\mathrm{eV}$, third $100-\mathrm{eV}$, and third $200-\mathrm{eV}$ events. The effect on the ambient distribution functions of firing the $\mathrm{Ar}^{+}$ beam is strongest during the first $100-\mathrm{eV}$ beam event (Figure $2 \mathrm{a}$ ), when the instrument payload was closest to the beamemitting subpayload. In this case, the distribution functions for both species are grossly modified with decreased amplitude and markedly broader extent in $V_{\text {perp }}$, indicative of intense perpendicular bulk heating. In subsequent $100-\mathrm{eV}$ beam firings, as shown in Figure $2 b$, the effect of the beam on the distributions is less extreme, although clear transverse heating of the $\mathrm{H}^{+}$is present. The $\mathrm{O}^{+}$is basically unaffected by the $100-\mathrm{eV}$ beam at these intermediate separations between the beam payload and the observation point. Finally, Figure $2 \mathrm{c}$ shows that at intermediate distances from the beam payload, the $200-\mathrm{eV}$ beam has only a minimal effect on the $\mathrm{H}^{+}$ distributions, with a slight enhancement of perpendicular velocities and, again, no measurable effect on the $\mathrm{O}^{+}$ions. At even larger separations from the injection point the effect of gun operations at either $100-\mathrm{eV}$ or $200-\mathrm{eV}$ upon the ambient ion distributions is minimal or nonexistent. Inspection of Plate 1 reveals that after $400 \mathrm{~s}$ TAL, corresponding to parallel and perpendicular payload separations of $600 \mathrm{~m}$ and $25 \mathrm{~m}$, respectively, no significant effect of the beam upon the measured ion temperatures was present.

\section{Electric Fields}

Measurements of electric fields were obtained during the ARCS-4 experiment, on board both the main and subpayloads. Low-frequency ac electric field observations obtained onboard the subpayload have been reported by Cahill et al. [1993]. Here we will present main payload observations obtained using the Cornell University instrumentation and comment on the observations of Cahill et al., as seems relevant.

Grey-scale frequency-time spectrograms of electric field fluctuations observed during four intervals during the flight are presented in Figure 3. The measurements shown in Figures 3 


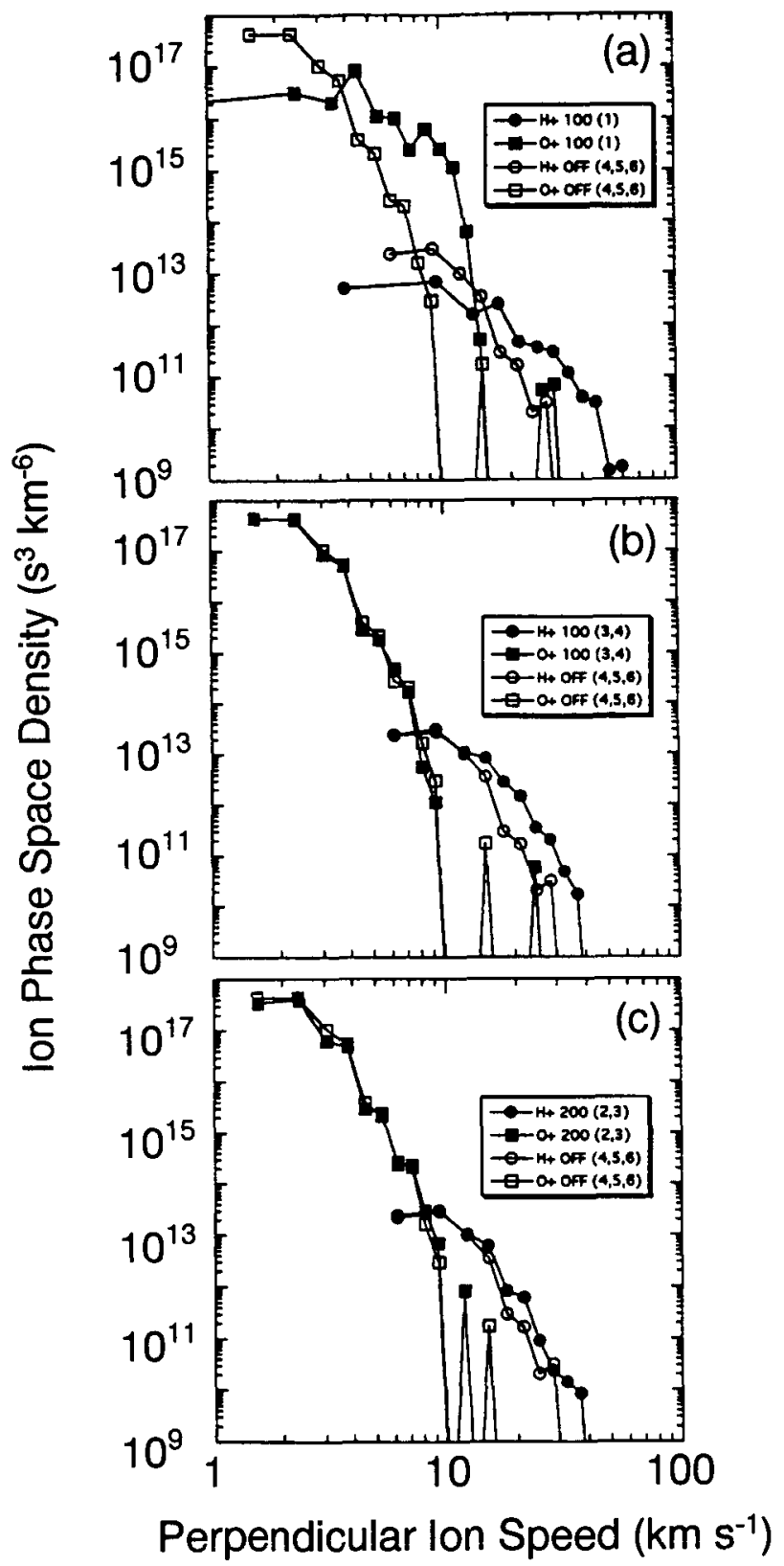

Figure 2. $\mathrm{H}^{+}$(circles) and $\mathrm{O}^{+}$(squares) ion phase space densities plotted versus $V_{\text {perp }}$ for beam-on (filled symbols) and beam-off (unfilled symbols) are displayed in each panel. The beam-off data are identical in each panel and represent an average of data obtained during intervals following the second $200-\mathrm{eV}$, third $100-\mathrm{eV}$, and third $200-\mathrm{eV}$ events. The beam-on data are from (a) the first $100-\mathrm{eV}$ beam event, (b) the third and fourth $100-\mathrm{eV}$ beam events, and (c) the second and third 200-eV beam events.

and 4 have not been scaled to account for the operation of the automatic gain control and are therefore presented in relative units. These data were obtained using the Cornell University 5.5-m Weitzmann boom antennas, deployed transverse to the main payload spin axis and therefore transverse to the geomagnetic field. The intervals shown include a portion of the first $100-\mathrm{eV}$ beam event (Figure $3 \mathrm{a}$ ), the second $200-\mathrm{eV}$ event (Figure $3 \mathrm{~b}$ ), a beam-off period between the second $200-\mathrm{eV}$ event and the third $100-\mathrm{eV}$ event (Figure $3 \mathrm{c}$ ), and the third
100 -eV event (Figure 3d). In each case, time is plotted along the abscissa, frequency $(0-16 \mathrm{kHz})$ along the ordinate, and the relative power spectral density is colored in grey scale as indicated by the bar at the bottom. Figure 3 illustrates that the electric field environment is stongly modified by the beam in a way which depends upon proximity to the beam source, the beam energy, and other factors, not well understood. The peri odic modulation of the wave power evident in Figures $3 a, 3 b$, and $3 \mathrm{~d}$ is at twice the rocket spin frequency, indicating it is due to alignment of the double probe antenna, rather than to a lighthouse effect as the beam sweeps with subpayload spin. Sudden changes in the wave spectra, such as that near $271 \mathrm{~s}$ TAL when power near $850 \mathrm{~Hz}$ and harmonics of that frequency appears, are notable features of the ARCS-4 wave observations and remain unexplained. Similar variability in the wave spectra was reported by Cahill et al. [1993].

In Figure 4 we show four electric field wave power spectra sampled from the intervals shown in Figure 3, at the times indicated. The features shown in these spectra are commonly observed during the first half of the flight, when beam-induced modifications of the ambient plasma were most evident at the main payload. The plotted points in Figure 4 are the actual 512 point fast Fourier transform (FFT) data, while the line is the result of applying a five-point smoothing function. In each panel, we have indicated the location of $\mathrm{H}^{+}$gyro harmonic (dashed vertical lines) and lower hybrid resonance (LHR) (bold vertical line) frequencies. The gyro harmonics are derived from the rocket position and a model (International Geomagnetic Reference Field (IGRF), 1980) magnetic field, while the LHR frequencies are determined from the model magnetic field and STICS ion species density measurements, in view of the cold plasma resonance condition [Stix, 1962] for a two-component $\mathrm{O}^{+}-\mathrm{H}^{+}$plasma. Each of the four power spectra shown has distinct characteristics which may be related to the ion spectra observed and presented above.

The spectrum in Figure $4 \mathrm{a}$ shows intense broadband wave emissions over the entire sampled frequency range, with no recognizable structure at either the $\mathrm{H}^{+}$gyro harmonics or the LHR frequency. Typical cross-field and field-aligned separations between the two payloads during this period were $3 \mathrm{~m}$ and $65 \mathrm{~m}$, respectively. The main payload was magnetically connected to a region approximately $3 \mathrm{~m}$ from the beam source, where the beam density was still quite high and coupling to the ambient plasma was extremely strong. The broadband waves at high intensity seen at this close distance are consistent with the predictions of Scales and Kintner [1990a, b] when the effects of finite parallel propagation are considered, as is the strong $\mathrm{H}^{+}$and $\mathrm{O}^{+}$heating. We note, however, that the electric field amplifier was in saturation during much of the interval displayed in Figure 3a and, in particular, during the interval displayed in Figure 4a. This may have masked features present in the real power spectrum.

The spectrum shown in Figure $4 \mathrm{~d}$ is also from a $100-\mathrm{eV}$ beam event but at a location more distant from the beam emitting payload. Broadband noise is again observed over the entire sampled frequency range but at a lower amplitude than that observed closer to the gun payload (Figure 4a). Although we have uncertainty regarding the states of the automatic gain control on the electric field wave receiver, we estimate that the energy density in these waves is more than 2 orders of magnitude smaller than that observed during the first $100-\mathrm{eV}$ event (Figure $4 \mathrm{a}$ ). The several intense spikes seen below the LHR frequency are suggestive of hydrogen gyro harmonic 

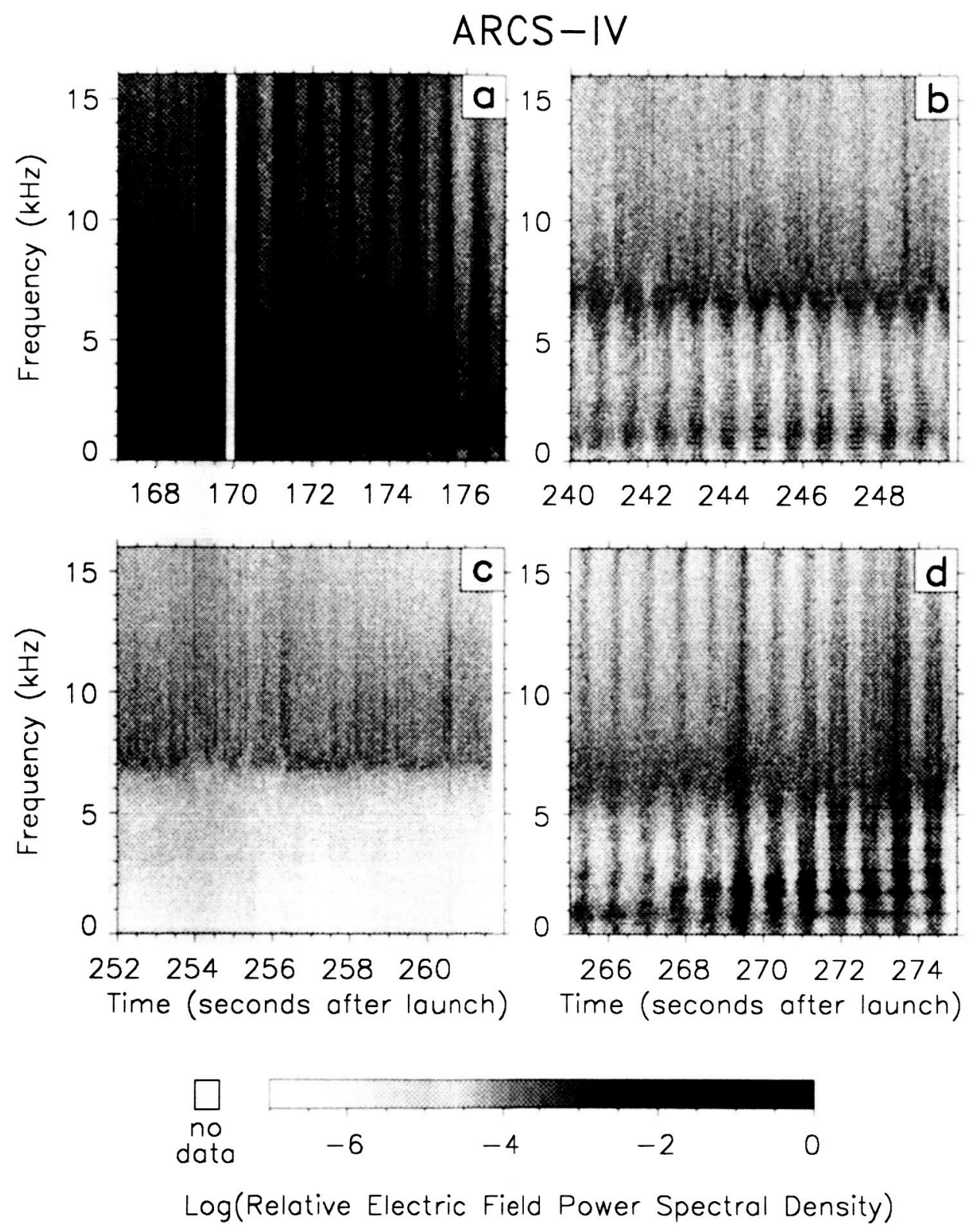

Figure 3. Frequency-time electric field spectrograms for four 10-s intervals during the ARCS-4 flight. These intervals include periods from (a) the first $100-\mathrm{eV}$ beam event, (b) the second $200-\mathrm{eV}$ event, (c) a beam-off period, and (d) the third 100-eV event. The data displayed are based on sliding 1024-point fast Fourier transforms (FFT) of measurements taken at intervals of $31.3 \mu \mathrm{s}$, providing a Nyquist frequency of $15.97 \mathrm{kHz}$ and a frequency resolution of $31.2 \mathrm{~Hz}$. The 1024-point FFT window is moved 512 points for each successive transform, providing a spectrum every $16 \mathrm{~ms}$. The data have not been corrected for automatic gain control function of the amplifier and are therefore plotted in relative units.

waves but are in fact at the wrong frequencies. These spikes are found at frequencies approximately equal to $\mathrm{N} * 880 \mathrm{~Hz}(1 \leq$ $\mathrm{N}$ ), while the $\mathrm{H}^{+}$gyrofrequency at this time is near $750 \mathrm{~Hz}$ in the $0.5 \mathrm{G}$ geomagnetic field at the rocket location. No reasonable value of geomagnetic field strength will yield a hydrogen gyrofrequency above $800 \mathrm{~Hz}$ at these altitudes. While it is tempting to assign this signature as due to hydrogen cyclotron harmonic waves, the fact that the spacing is so large remains unexplained.

The spectrum in Figure $4 \mathrm{c}$ is from a beam-off period and shows naturally occurring auroral hiss [Bering et al., 1987], characterized by maximum power near the LHR frequency and severe attenuation below. Very little naturally occurring electric field wave power is observed in the low-frequency portion of the spectrum. Finally, Figure $4 \mathrm{~b}$ shows the power spectrum of waves observed during a $200-\mathrm{eV}$ beam event. Here, the broadband component of the spectrum looks similar to that of that of Figure $4 \mathrm{~d}$ for the $100-\mathrm{eV}$ case but without the strong enhancements at multiples of $880 \mathrm{~Hz}$. Weaker harmonic structure is seen in this case, with peaks spaced by $264 \mathrm{~Hz}$. The energy density in this spectrum is roughly an order of magnitude smaller than that in the spectrum of Figure $4 \mathrm{~d}$. 


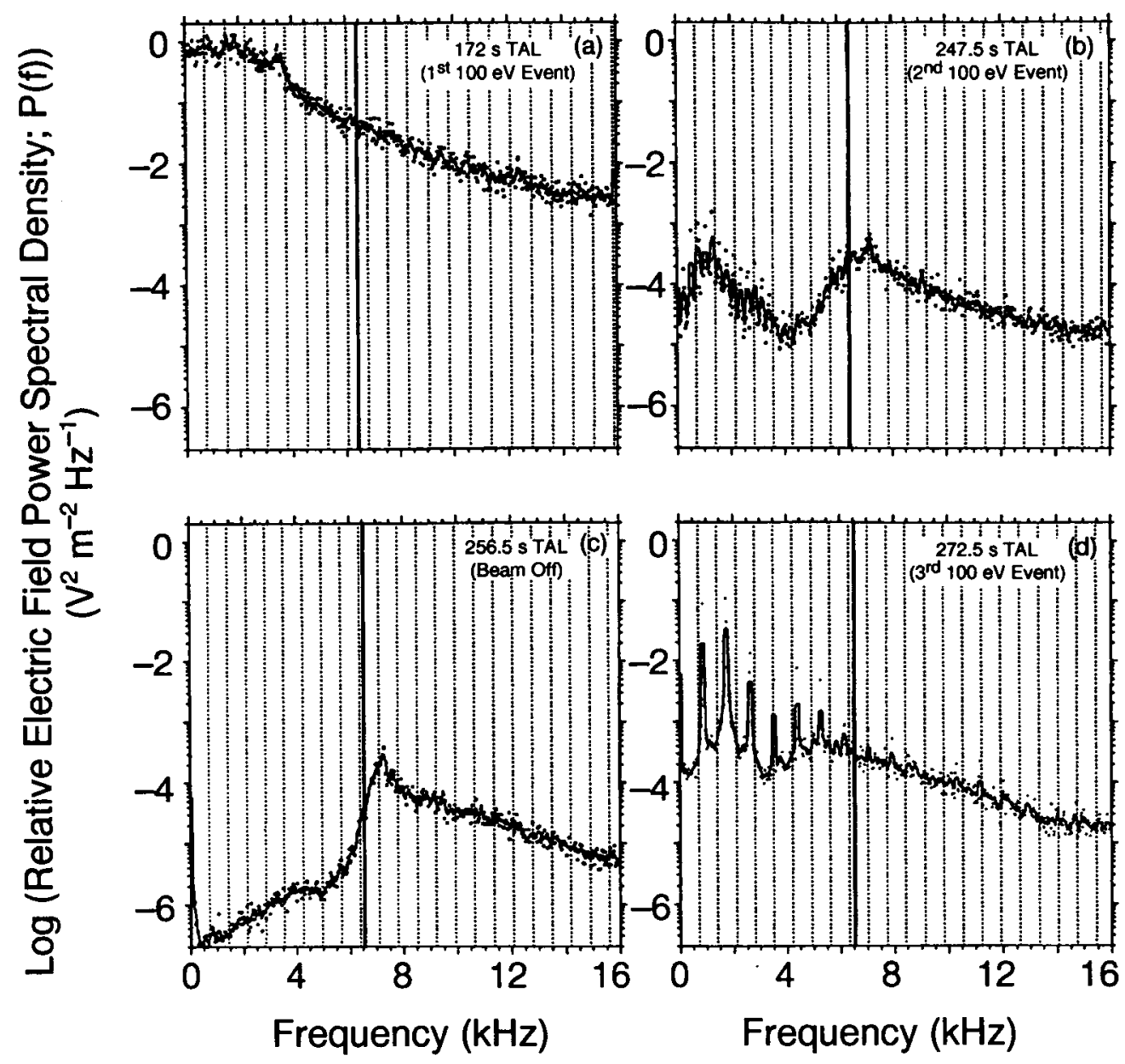

Figure 4. Individual power spectra of electric field fluctuations at four selected times during the ARCS-4 experiment, plotted in relative units, as in Figure 3. Each spectrum results from averaging 31 ( $0.5 \mathrm{~s}$ of data) power spectra together. The four times shown are selected from the four spectrograms presented in Figure 3 and correspond to intervals during (a) the first $100-\mathrm{eV}$ beam event, (b) the second 200-eV event, (c) a beam-off period, and (d) the third $100-\mathrm{eV}$ event. The dashed vertical lines are at the expected frequencies of $\mathrm{H}^{+}$gyro harmonics, and the bold vertical line in each panel is located at the local ambient lower hybrid frequency.

\section{Discussion}

We have presented ion distribution function and electric field wave observations obtained on the ARCS-4 main payload in the upper $F$ region ionosphere, in the vicinity of an $\mathrm{Ar}^{+}$ion-emitting source located on the ARCS-4 subpayload. The two payloads moved apart from each other as the experiment proceeded, allowing measurements to be made at varying distances from the ion source. Although the relative motion of the two payloads was primarily along the local magnetic field, there was a cross-field component to the motion. The 100- and 200-eV ion beams emitted from the subpayload were directed across field lines. At any given time, the instrumented main payload sampled field lines which threaded the beam itself at a well-defined, cross-field distance from its source. Although no direct measurements of the ARCS- 4 beam profile have been performed, measurements of sources of similar design flown on previous ARCS payloads have shown the beam to carry roughly $100 \mathrm{~mA}$ into a $30^{\circ}$ (half angle) conical volume centered on the look direction of the source [Erlandson, 1984]. These measurements also show the beam ion temperature close to the source to be of the order of
$50 \mathrm{eV}$. Using these numbers and neglecting the magnetic curvature of the beam ion trajectories, we may estimate the beam density, drift, and thermal velocity as functions of cross field distance from the source. In conjunction with STICS measurements of the background plasma densities, an estimate of the Scales and Kintner [1990a] beam plasma coupling parameter $\left(\tau^{2}\right)$ is provided (see (1)) in an attempt to order the particle and wave observations. Such an estimate is shown in Figure 5. Here we have plotted the coupling parameter versus ARCS-4 time after launch for the $100-\mathrm{eV}$ and $200-\mathrm{eV}$ beam cases. We have also indicated on the plot the occurrence of $100-\mathrm{eV}$ and $200-\mathrm{eV}$ ion beam firings for the first few events, including those for which data have been presented above.

From Figure 5, we see that the value of $\tau^{2}$ varied over the range of interest (from large to small, compared to 1 ) over the early course of the experiment. During the first $100-\mathrm{eV}$ beam event, its value was large, decreasing from near 60 at the beginning of the event to near 10 when the gun was turned off. This is the period when main payload observations included heating of both the ambient $\mathrm{O}^{+}$and $\mathrm{H}^{+}$ions to near $3 \mathrm{eV}$ and the extremely intense emission of broadband electric field waves over the entire $16-\mathrm{kHz}$ frequency range was observed. 


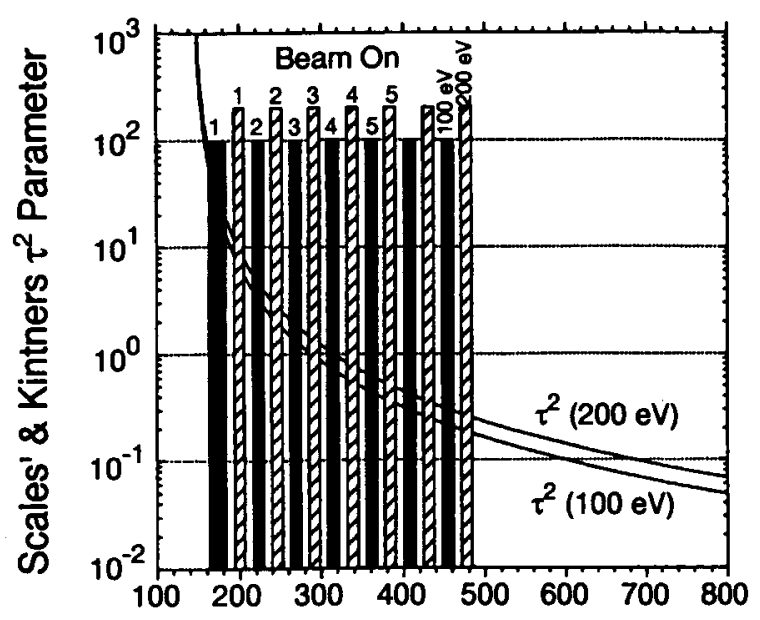

ARCS IV Time After Launch (s)

Figure 5. Beam plasma coupling parameter $\left(\tau^{2}\right)$ plotted as a function of ARCS-4 time after launch for the 100-eV (lower curve) and 200-eV (upper curve) cases. The times when the argon ion beam was operating in either the $100-\mathrm{eV}$ or $200-\mathrm{eV}$ mode are indicated for the first seven of these events. The shaded vertical bars indicate $100-\mathrm{eV}$ beam-on times, and the hatched vertical bars indicate $200-\mathrm{eV}$ beam-on times. The numbers at the top of the bars indicate which beam event is in progress, for example, the second $100-\mathrm{eV}$ event in progress between approximately 240 and $252 \mathrm{~s}$ after launch.

This is the only event in which the $\mathrm{O}^{+}$plasma component was affected by the beam. During this period, the broadband wave power was at least 2 to 3 orders of magnitude larger than at any other time during the experiment, providing electric field amplitudes large enough to trap the thermal $\mathrm{O}^{+}$ions in the associated potential wells. These observations are consistent with the predictions of Scales and Kintner [1990a, b].

Subsequent 100 -eV events, through the fifth, produced heating, primarily of the $\mathrm{H}^{+}$ions, as well as broadband wave emissions at distinctly lower amplitudes than the first $100-\mathrm{eV}$ event. Large amplitude harmonic emissions, similar to those shown in Figures 3d and 4d, were observed sporadically through the first half of the flight during $100-\mathrm{eV}$ events and occasionally during $200-\mathrm{eV}$ events. However, these harmonic emissions were sporadic enough that they are not believed to be responsible for the observed $\mathrm{H}^{+}$heating. Rather, the broadband waves, which were always present during early events, are believed to be the responsible heating agent. After the fifth $100-\mathrm{eV}$ beam event the value of the coupling parameter dropped below 0.4 and no further heating of either species was observed, although broadband waves, over the entire $16 \mathrm{kHz}$ experiment frequency range, were observed at the main payload during $100-\mathrm{eV}$ events throughout the flight.

In the case of the $200-\mathrm{eV} \mathrm{Ar}^{+}$beam, only during the first of these events was heating of the $\mathrm{H}^{+}$ions observed to be comparable to that induced by the $100-\mathrm{eV}$ beam injections. We note (Platele) that small temperature enhancements were seen in the $\mathrm{H}^{+}$during the second through fourth firing of the $200-\mathrm{eV} \mathrm{Ar}{ }^{+}$beam. In no case did the $200-\mathrm{eV} \mathrm{Ar}^{+}$injections measurably perturb the background $\mathrm{O}^{+}$distributions. These effects were predicted by Scales and Kintner [1990a, b] and are understood in terms of proximity to resonance (or lack thereof) through matching of the injected $\mathrm{Ar}^{+}$ions' drift speed with the ambient species thermal speed, via the phase speed of destabilized waves. In the current case, taking $0.5 \mathrm{eV}$ ambient perpendicular ion temperatures, the argon ion drift speed is in the ratio of 3:1 and 2:1 compared to the $\mathrm{H}^{+}$thermal speed and 13:1 and 9:1 compared to the $\mathrm{O}^{+}$thermal speed for the 200- and 100-eV beams, respectively. Given the large $\mathrm{Ar}^{+}$ beam temperature, there are a substantial number of $\mathrm{Ar}^{+}$ions that satisfy a speed matching condition with thermal $\mathrm{H}^{+}$ions, particularly for the $100-\mathrm{eV}$ case, and relatively few which match the much slower $\mathrm{O}^{+}$thermal speed.

The value of the coupling parameter for the $200-\mathrm{eV}$ beam exceeds that for a $100-\mathrm{eV}$ beam at any given time. Nominal values of the coupling parameter at the observation times for the two spectra shown in Figures $4 \mathrm{~b}$ and $4 \mathrm{~d}$ were 1.3 for the 100-eV beam (Figure $4 \mathrm{~d}$ ) and 2.8 for the $200-\mathrm{eV}$ beam (Figure 4b). Both cases resulted in broadband wave excitation and harmonic structure, as described above. Later in the flight, the $100-\mathrm{eV}$ events continued to produce broadband waves, although these eventually became limited to frequencies near and above the lower hybrid frequency and extended to the 16 $\mathrm{kHz}$ experiment Nyquist frequency. The waves produced by later $200-\mathrm{eV}$ events took on a much more distinctly narrowband character with emissions limited to the vicinity of the lower hybrid frequency.

\section{Conclusions}

On the basis of analysis of the distribution functions of $\mathrm{O}^{+}$ and $\mathrm{H}^{+}$and the electric field frequency spectra, all measured at the ARCS-4 main payload as it moved away from the subpayload, we have been able to draw several conclusions regarding the modification of the local plasma environment near the beam-emitting subpayload. These include the following:

1. Very near the subpayload, bulk heating of both ion species (to the order of $3 \mathrm{eV}$ ) occurs during $100-\mathrm{eV} \mathrm{Ar}^{+}$beam injections. During this interval, the beam-plasma coupling parameter of Scales and Kintner [1990a] is large (>10). The ion heating is due to trapping in the large amplitude waves observed over the entire $(0-16 \mathrm{kHz})$ frequency range of the electric field experiment, as predicted by Scales and Kintner [1990b].

2. During subsequent beam events, only the $\mathbf{H}^{+}$ions are observed to be heated and these are heated more strongly during $100-\mathrm{eV}$ events than during $200-\mathrm{eV}$ events. The $\mathrm{O}^{+}$ions are not measurably affected by beam injections after the first event. In these cases, the heating is again due to trapping in potential wells associated with the observed broadband waves. The $\mathrm{O}^{+}$is not heated because in the frame of reference of the propagating waves the kinetic energy of the $\mathrm{O}^{+}$is too large to allow trapping and energization.

3. The $100-\mathrm{eV}$ beam events couple energy more effectively into the $\mathrm{H}^{+}$ions than the $200-\mathrm{eV}$ events because the drift speed of the $100-\mathrm{eV} \mathrm{Ar}^{+}$ions $\left(22 \mathrm{~km} \mathrm{~s}^{-1}\right)$ more closely matches the transverse thermal speed of the $\mathrm{H}^{+}$ions $(9.8 \mathrm{~km}$ $\mathrm{s}^{-1}$ ) than does the drift speed of the $200-\mathrm{eV} \mathrm{Ar}^{+}$ions $(31 \mathrm{~km}$ $\left.s^{-1}\right)$.

Acknowledgments. Development of STICS was engineered and managed by W. L. Chisholm of MSFC. Its magnetic mass analyzer is based on that developed for the Dynamics Explorer 1 Retarding Ion Mass Spectrometer by C. R. Chappell, D. L. Reasoner, and S. A. 
Fields of MSFC with J. H. Hoffman and W. W. Wright of the University of Texas at Dallas. The STICS electrostatic optics were raytraced and optimized by V. N. Coffey and P. Sloan of MSFC. The STICS sequencer and low voltage power supplies were developed by J. Currie and P. Smith of MSFC. The STICS stepping power supplies were developed by $W$. W. Wright and D. Tipton of the University of Texas at Dallas. V. N. Coffey documented the response characteristics of the STICS flight model. Preparation of graphics and assistance with data displays were provided by P. Sloan of MSFC. This work was supported by the OSSA Space Physics Division, Ionospheric Physics Branch, Suborbital Program under UPNs 442-36 and 435-11.

The Editor thanks J. H. Hoffman and G. Haerendel for their assistance in evaluating this paper.

\section{References}

Banks, P. M., and T. E. Holzer, High-latitude plasma transport: The polar wind, J. Geophys. Res., 74, 6317, 1969.

Bering, E. A., J. E. Maggs, and H. R. Anderson, The plasma environment of an auroral arc, 3, VLF hiss, J. Geophys. Res., 92, 7581, 1987.

Cahill, L. J., Jr., R. L. Amoldy, R. L. Lysak, W. Peria, and K. A. Lynch, Waves generated in the vicinity of an argon plasma gun in the ionosphere, J. Geophys. Res., 98, 9483, 1993.

Eriandson, R. E., Design and test of a plasma beam for space plasma studies, M.S. thesis, Univ. of Minn., Minneapolis, 1984.

Erlandson, R. E., Plasma waves generated during an artificial ion beam experiment in the ionosphere, Ph.D. thesis, Univ. of Minn., Minneapolis, 1986.

Erlandson, R. E., L. J. Cahill Jr., C. J. Pollock, R. L. Arnoldy, W. A. Scales, and P. M. Kintner, Initial results from the operation of two argon ion generators in the auroral ionosphere, J. Geophys. Res., 92, $4601,1987$.

Erlandson, R. E., L. J. Cahill Jr., R. L. Kaufmann, C. J. Pollock, and R. L. Arnoldy, ARCS 3 ionospheric artificial argon ion beam injections: Waves near the heavy ion gyrofrequencies, J. Geophys. Res., 94, 2645, 1989.

Haerendel, G., and R. Z. Sagdeev, Artificial plasma jet in the ionosphere, Adv. Space Res., 1, 29, 1981.

Hăusler, B., R. A. Treumann, O. H. Bauer, G. Haerendel, R. Bush, C. W. Carlson, B. Theile, M. C. Kelley, V. S. Dokukin, and Y. Y.
Ruzhin, Observations of the artificially injected Porcupine xenon ion beam in the ionosphere, J. Geophys. Res., 91, 287, 1986.

Jones, $\mathrm{D} ., \mathrm{Xe}^{+}$-induced ion-cyclotron harmonic waves, Adv. Space Res., $1,103,1981$.

Kaufmann, R. L., D. N. Walker, J. C. Holmes, C. J. Pollock, R. L. Arnoldy, L. J. Cahill Jr., and P. M. Kintner, Heavy ion beamionosphere interactions: Charging and neutralizing the payload, $J$. Geophys. Res., 94, 4523, 1989.

Kintner, P. M., and M.C. Kelley, Ion beam produced plasma waves observed by the $\Delta \mathbf{n} / \mathbf{n}$ plasma wave receiver during the Porcupine experiment, Adv. Space Res., 1, 107, 1981.

Klumpar, D. M., Transversely accelerated ions: An ionospheric source of hot magnetospheric ions, J. Geophys. Res., 84, 4229, 1979.

Pollock, C. J., Rocket-borne low energy ion measurements in space, Ph.D. thesis, Univ. of New Hampshire, Durham, 1987.

Pollock, C. J., R. L. Amoldy, R. E. Erlandson, and L. J. Cahill Jr., Observations of the plasma environment during an active ionospheric ion beam, J. Geophys. Res., 93, 11,473, 1988.

Scales, W. A., and P. M. Kintner, Artificial ion beam instabilities, 1, Linear theory, J. Geophys. Res., 95, 10,623, 1990a.

Scales, W. A., and P. M. Kintner, Artificial ion beam instabilities, 2, Simulations, J. Geophys. Res., 95, 10,643, 1990b.

Sharp, R. D., R. G. Johnson, and E. G. Shelley, Observation of an ionospheric acceleration mechanism producing energetic (keV) ions primarily normal to the geomagnetic field direction, $J$. Geophys. Res., 82, 3324, 1977.

Stix, T. H., The Theory of Plasma Waves, McGraw-Hill, New York, 1962.

R. L. Amoldy, Institute for the Study of Earth, Oceans, and Space, University of New Hampshire, Durham, NH 03824.

L. J. Cahill Jr., School of Physics and Astronomy, University of Minnesota, Minneapolis, MN 55455.

M. O. Chandler, T. E. Moore, and C. J. Pollock, Space Plasma Physics Branch, Space Sciences Laboratory, NASA Marshall Space Flight Center, Huntsville, AL 35812. (e-mail: mooret@mpb.msfc.nasa. gov; chandler@mpb.msfc.nasa.gov; pollock@mpb.msfc.nasa.gov)

S. Chesney and P. M. Kintner, Department of Electrical Engineering, Cornell University, Ithaca, NY 14850.

(Received August 12, 1994; revised December 9, 1994; accepted December 9, 1994.) 